\title{
Cuidados de enfermagem ao paciente submetido à litotripsia extracorpórea em centro cirúrgico ambulatorial
}

\author{
Nursing care for patients undergoing extracorporeal lithotripsy in the ambulatory surgical \\ center
}

\author{
Atención de enfermería al paciente sometido a litotricia extracorpórea en un centro \\ quirúrgico ambulatorial
}

\begin{abstract}
Débora Machado Nascimento do Espírito Santo ${ }^{1}$, Lisiane Paula Sordi Matzenbacher ${ }^{1}$, Rosaura
\end{abstract} Soares Paczek ${ }^{1 *}$, Carina Galvan ${ }^{1}$, Ana Karina Silva da Rocha Tanaka².

\section{RESUMO}

Objetivo: Descrever os cuidados prestados pela equipe de Enfermagem ao paciente submetido à litotripsia extracorpórea. Relato de experiência: Trata-se de um procedimento minimamente invasivo para o tratamento da litíase renal. Com baixa incidência de complicações, sendo primeira opção de tratamento, realizada normalmente sob anestesia, em nível ambulatorial, dura aproximadamente 40 minutos, pode ser dolorosa, dependendo da intensidade, escalonamento gradual de tensão e número de impulsos aplicados. $\mathrm{Na}$ admissão a equipe de Enfermagem, confirma o jejum, verifica os sinais vitais, coloca a pulseira de identificação, realiza a anamnese e presta assistência durante todo o procedimento. As orientações de alta hospitalar são realizadas pela enfermeira da sala de recuperação. Pode ocorrer cólicas, dor lombar ou em região de baixo ventre e discreto sangramento urinário; é usual aparecer algumas manchas roxas ou avermelhadas no local. Estimula-se a ingestão de líquidos, com liberação dieta e manutenção de repouso relativo no dia do exame. Considerações finais: Os cuidados de Enfermagem contemplam todas as necessidades do paciente, respeitando suas individualidades e atentando-se às possíveis complicações, prestando uma assistência de qualidade durante a permanência dos pacientes do preparo à sala de recuperação anestésica.

Palavras-chave: Cuidados de enfermagem, Litotripsia, Cálculos urinários.

\begin{abstract}
Objective: To describe the care provided by the Nursing team to patients undergoing extracorporeal lithotripsy. Experience report: This is a minimally invasive procedure for the treatment of renal lithiasis. With low incidence of complications, it is the first option for treatment, usually performed under anesthesia, at outpatient level, it lasts approximately 40 minutes, may be painful, depending on the intensity, gradual escalation of tension and number of impulses applied. On admission, the Nursing team confirms fasting, checks vital signs, puts on the identification bracelet, performs the anamnesis, and provides assistance throughout the procedure. Discharge instructions are given by the nurse in the recovery room. There may be cramps, pain in the lower back or lower abdomen, slight urinary bleeding, and it is usual for some purple or reddish spots to appear at the site. Fluid intake is encouraged, with dietary release and maintenance of relative rest on the day of the exam. Final considerations: The nursing care contemplates all the patient's needs, respecting their individuality and paying attention to possible complications, providing quality assistance during the patients's stay from the preparation to the anesthesia recovery room.
\end{abstract}

Keywords: Nursing care, Lithotripsy, Urinary calculi.

${ }^{1}$ Hospital de Clínicas de Porto Alegre, Porto Alegre - RS. *E-mail: rspaczek@gmail.com

2 Universidade Federal do Rio Grande do Sul, Porto Alegre - RS. 


\section{RESUMEN}

Objetivo: Describir la atención brindada por el equipo de Enfermería a los pacientes sometidos a litotricia extracorpórea. Informe de experiencia: Se trata de un procedimiento mínimamente invasivo para el tratamiento de cálculos renales. Con una baja incidencia de complicaciones, siendo la primera opción de tratamiento, generalmente realizada bajo anestesia, a nivel ambulatorio, tiene una duración aproximada de 40 minutos, puede ser dolorosa, dependiendo de la intensidad, escalada gradual de tensión y número de impulsos aplicados. Al ingreso, el equipo de Enfermería confirma el ayuno, revisa los signos vitales, se pone la pulsera de identificación, realiza la anamnesis y brinda asistencia durante todo el procedimiento. La enfermera lleva a cabo las pautas de alta en la sala de recuperación. Puede haber calambres, lumbalgia o dolor en la parte baja del abdomen, sangrado urinario leve, es habitual que aparezcan algunas manchas violáceas o rojizas en la zona. Se fomenta la ingestión de líquidos, con liberación de dieta y mantenimiento de reposo relativo el día del examen. Consideraciones finales: La atención de Enfermería cubre todas las necesidades del paciente, respetando su individualidad y prestando atención a las posibles complicaciones, brindando una atención de calidad durante la estadía de los pacientes en preparación para la sala de recuperación anestésica.

Palabras clave: Atención de enfermería, Litotricia, Cálculos urinarios.

\section{INTRODUÇÃO}

A litíase urinária é uma condição patológica bastante frequente, cuja prevalência pode alcançar até $20 \%$ da população mundial, sendo maior em países de clima quente, provocando gastos significativos nos sistemas de saúde. Com a criação de instrumentos endourológicos e considerando a ampla capacidade dos aparelhos utilizados, criaram-se condições para que apenas $10 \%$ das cirurgias possam ser realizadas de forma aberta (RODRIGUEZ GY, et al., 2019; KIM JK, et al., 2016).

A litotripsia extracorpórea é um tratamento utilizado por médicos urologistas para a abordagem terapêutica de cálculos renais e urinários por meio de ondas de choque (LECO). Trata-se de um procedimento minimamente invasivo para o tratamento da litíase renal com baixa incidência de complicações e utilizado como primeira opção de tratamento (BERBER-DESEUSA A, et al., 2017). São empregadas ondas sonoras e mecânicas, de alta potência de choque, criadas e transmitidas por um aparelho chamado litotritor, emitidas a distância que se propaga em meio líquido e penetram na região que abriga os cálculos por vibração, obtendo seu esmagamento ou trituração e, consequentemente, induzindo sua eliminação completa ou fragmentação. O objetivo é reduzi-los a fragmentos menores, passíveis de eliminação espontânea, sendo os cálculos localizados através de um exame de radiologia abdominal (TURK C, et al., 2017; ALVES NG, et al., 2020).

Em razão disso, é considerada a primeira opção de tratamento em pacientes pediátricos, apresentando resultados melhores do que os evidenciados em adultos. Isto se deve ao menor tamanho, à fragilidade dos cálculos e ao peso corporal inferior, o que facilita a propagação das ondas de choque devido à maior proporção de água e maior elasticidade dos tecidos e ao maior peristaltismo dos canais uretrais, propiciando a expulsão dos cálculos (RODRíGUEZ GY, et al., 2019).

Possíveis complicações podem ser decorrentes desse tipo de tratamento, como os hematomas renais. Além disso, podem permanecer fragmentos dos cálculos alojados nos rins ou nas vias urinárias, causando dor, cólica renal ou ferindo os ureteres, trombose nas veias ilíacas e porta e a mais catastrófica de todas e potencialmente fatal: o rompimento de aneurisma de aorta abdominal. A litotripsia tem ainda como principal contraindicação a gravidez (FORNAZARI VAV, et al., 2021).

Todos os pacientes precisam de cuidados pré e pós-operatórios por parte do enfermeiro para prevenir complicações e intervenções decorrentes do exame. $O$ enfermeiro deve enfatizar ao paciente a importância de relatar os sintomas imediatamente. Os cuidados pós-operatórios devem incluir: monitoramento rigoroso dos sinais vitais, observação cuidadosa da diurese espontânea, cuidados com o curativo e prevenção de infecção (ABDELMOWLA RAA, et al., 2017). 
Para que o cuidado de enfermagem no transoperatório seja seguro e de qualidade, faz-se necessária a Sistematização da Assistência de Enfermagem (SAE), buscando uma atuação mais participativa do enfermeiro, sendo este o responsável por garantir a segurança nos processos relacionados a admissão do paciente, ao posicionamento cirúrgico, assim como certificar-se da esterilização dos materiais necessários e a disponibilidade de itens essenciais de farmácia (JOST MT, et al., 2019).

A equipe de enfermagem acompanha o paciente durante todo o perioperatório, sendo um facilitador na percepção de aflições e incertezas. É uma vivência cirúrgica onde se faz presente a prática humanizada dos profissionais de enfermagem, tanto nos processos internos a que o paciente é submetido, quanto na orientação de alta hospitalar. O paciente deve possuir autonomia para que, desta forma, sinta-se seguro, demonstrando capacidade de dominar a situação vivenciada (SOUZA IB, et al., 2019).

As orientações e cuidados de enfermagem são essenciais desde a admissão até a alta do paciente, pois auxilia no nível de conhecimento e tem efeito significativo na redução ou prevenção de complicações pósoperatórias e na melhoria da qualidade de vida (ABDELMOWLA RAA, et al., 2017).

Este relato de experiência tem o objetivo de descrever a prática vivida de um grupo de enfermeiras de um centro cirúrgico ambulatorial onde se encontra uma sala exclusiva para a realização de LECO. O propósito deste trabalho é colaborar com a construção de conhecimento e o aperfeiçoamento da sistematização da assistência de Enfermagem perioperatória.

\section{RELATO DA EXPERIÊNCIA}

A LECO é realizada, na maioria das vezes, em nível ambulatorial. O procedimento é agendado com antecedência e, no dia e horário marcados, o paciente comparece ao serviço de centro cirúrgico. Após realizar o cadastro na secretaria e a conferência do termo de consentimento informado do procedimento, que deverá estar assinado pelo médico e pelo paciente e/ou responsável, o mesmo é encaminhado para a sala de preparo onde é recebido pela equipe de Enfermagem. Será confirmado o tempo de jejum, verificados os sinais vitais, colocada a pulseira de identificação e realizada a anamnese pela enfermeira com a finalidade de se obterem informações do processo saúde-doença e de se identificarem os problemas existentes.

Em seguida, o paciente é orientado a trocar de roupa e a retirar os adornos, vestindo a camisola padrão institucional para aguardar na sala de preparo onde o médico anestesista realiza a avaliação pré-anestésica, aplicando o termo de consentimento informado da anestesia/sedação. O técnico de Enfermagem responsável pela sala de procedimento chama o paciente pelo nome completo, verificando-o na escala de procedimentos. Após a conferência da pulseira de identificação, o paciente é encaminhado para a realização da LECO. O procedimento é executado por médicos urologistas, que operam e monitoram o equipamento, acompanhados pelo anestesiologista e pela equipe de Enfermagem, que realiza o preparo da sala, o acompanhamento, a orientação, a punção venosa e a administração de medicação.

A LECO dura aproximadamente 40 minutos e pode ser dolorosa, dependendo do tipo de máquina empregado, da intensidade, do escalonamento gradual de tensão e do número de impulsos aplicados. Pode ser realizada sob anestesia do tipo sedação, que auxilia diminuindo a sensibilidade dolorosa e provocando o relaxamento do paciente, mantendo ou não a sua consciência, permitindo as condições para ser utilizada mais energia de choque.

Durante o procedimento, o paciente é posicionado sobre a mesa do equipamento em decúbito dorsal e monitorizado com sinais vitais (oximetria, pressão arterial não invasiva, frequência cardíaca e frequência respiratória). Realiza-se, então, a punção de acesso periférico, instalando-se a soroterapia conforme avaliação médica. Após a sedação, o paciente é posicionado adequadamente, sendo realizada a fluoroscopia para a localização do cálculo.

Ainda durante o procedimento, toda a equipe presente deverá utilizar os equipamentos de proteção individual, como o avental de chumbo e o protetor de tireoide. A sala para a realização do procedimento é equipada com o equipamento litotritor e o aparelho de anestesia e deve ser montada com todo material para manter uma via aérea permeável, como máscara facial, tubo orotraqueal e guedel. 
Após o procedimento, o paciente ficará em sala de recuperação pós-anestésica em observação, por um período mínimo de aproximadamente uma hora, até recuperar-se da anestesia. Os sinais vitais são verificados a cada 15 minutos na primeira hora, de $30 \mathrm{em} 30$ minutos na segunda hora e de hora em hora nos horários subsequentes e a alta hospitalar ocorre após a reavaliação médica. O paciente precisa estar bem recuperado da anestesia, com volume de diurese espontânea, no mínimo, de $100 \mathrm{ml}$, e esta deverá ser avaliada quanto à coloração e característica.

Podem ocorrer pequenas escoriações na pele no local do procedimento e compete à equipe de Enfermagem identificar e inspecionar a pele antes da alta e, em caso de lesões, avaliar e, se necessário, realizar curativo simples. Dentro de poucos dias, essas escoriações cicatrizam e, neste contexto, deve-se ter a garantia da qualidade e segurança do paciente como foco principal.

As orientações de alta hospitalar relacionadas aos cuidados pós-procedimento no domicílio são realizadas pela enfermeira da sala de recuperação. Os pacientes podem apresentar cólicas, dor lombar ou em região de baixo ventre e discreto sangramento urinário com a presença de alguns pequenos coágulos. Podem aparecer também algumas manchas roxas ou avermelhadas no local onde foi realizada a LECO. Os pacientes são estimulados à ingestão de líquidos, como água, sucos naturais ou chás, e é liberada uma dieta leve via oral, mantendo o repouso relativo no dia do exame.

Os pacientes são ainda orientados quanto ao retorno do uso da medicação de uso contínuo, se necessário, e a evitar esforços físicos intensos enquanto a urina estiver hematúria. Geralmente, a urina retorna à coloração normal dentro de dois ou três dias e, caso não se consiga colher algum fragmento dos cálculos, deve-se filtrar a diurese espontânea no domicílio com coador de pano para observar se eles foram expelidos. Pode não ocorrer a fragmentação completa dos cálculos renais, por isso, deve-se agendar exame radiológico de abdome, conforme solicitado pela equipe médica, para o monitoramento deles.

No momento da alta hospitalar, o paciente deve estar acompanhado de um adulto e é orientado a comparecer à emergência caso apresente: dor forte, que não melhore com os medicamentos prescritos; sangramento ativo uretral; dor ao urinar; retenção urinária; febre ou sintoma de mal-estar. São entregues, ao paciente, todas as orientações citadas acima em formulário padrão institucional juntamente com o retorno para consulta, que deverá ser nas próximas três a quatro semanas, além da solicitação do exame radiológico.

\section{DISCUSSÃO}

O centro cirúrgico é considerado um ambiente de alto risco por realizar procedimentos complexos onde acontece grande parte dos eventos adversos à saúde, relações interdisciplinares, culturas distintas e trabalho sob pressão. Podem ocorrer danos reversíveis ou irreversíveis relacionados à cirurgia e anestesia. Assim, 0 foco principal é a qualidade da assistência e cuidados ao paciente, contribuindo com a prevenção de agravos à integridade física (ACOSTA AM, et al., 2018; FASSARELLA CS, et al., 2020; HENRIQUES AHB, et al., 2016).

O paciente é o foco da assistência e as equipes de saúde e de Enfermagem são responsáveis pelos seus cuidados desde o pré-operatório até a recuperação anestésica. O enfermeiro necessita de uma comunicação efetiva com o paciente e familiar, evitando informações divergentes e incorretas, proporcionando conforto e minimizando dúvidas, ansiedade e outros sentimentos que podem surgir desde a chegada até a alta hospitalar (CAVERZAN TCR, et al., 2017; SOBECC, 2017; GUTIERRES LS, et al., 2018).

Dentro das variáveis que podem ser modificadas durante a realização do procedimento, encontram-se a frequência do disparo e o acoplamento adequado entre paciente e equipamento (CONTRERAS PN, 2018). O procedimento deve iniciar-se com baixa energia, sendo aumentada gradualmente. As ondas de choque sucessivas provocam cisalhamento, assim como disposições de bolhas de cavitação em torno dos cálculos, que se rompem, potencializando a sua desintegração (TORRICELLI FCM, et al., 2015).

Os cálculos com dois centímetros são indicados para tratamento percutâneo. O tamanho do cálculo influencia a taxa de sucesso e, por vezes, a sua fragmentação não ocorre, necessitando de procedimentos complementares (FELICI EM, et al., 2017). Estudos apontam preditores de sucesso e pontos ideais para o emprego desta técnica, sendo: densidade do cálculo; diâmetro da litíase; índice de massa corporal; distância da pele à pedra, assim como a sua localização (BENGIO RG, et al., 2016; YAZICI O, et al., 2015). 
O procedimento apresenta baixa taxa de complicações, porém, existe a necessidade de reaplicação e da realização de procedimentos complementares. A posição inicial do paciente depende da localização do cálculo e do índice de massa corporal do paciente. Podem ocorrer arritmias cardíacas, como a taquicardia ventricular, devido à estimulação cardíaca pelas ondas de choque e, em caso de arritmia sustentada, o tratamento deve ser suspenso por dois minutos e, em seguida, reiniciado. Se o paciente desenvolver novamente uma arritmia, ele será girado $15^{\circ}$ a $20^{\circ}$ da posição original, estratégia que pode eliminar a arritmia, e o tratamento será reiniciado (FELICI EM, et al., 2017).

Dessa forma, para que o paciente se sinta seguro em um ambiente cirúrgico, é imprescindível a implantação da sistematização da assistência de Enfermagem, visando ao cuidado individualizado e específico às necessidades de cada paciente. Cabe à Enfermagem transmitir segurança e confiança, minimizando a angústia e ansiedade, sentimentos normais nesse ambiente desconhecido (CASTANHEIRA JS, et al., 2020).

A limitação deste estudo refere-se a ter sido realizado somente em uma instituição hospitalar da região Sul do Brasil. Identificou-se a escassez de estudos que evidenciem a atribuição do enfermeiro no pré, trans e pós-procedimento de litotripsia extracorpórea.

A equipe de Enfermagem é peça fundamental por conhecer toda a complexidade das fases no pré, trans e pós-procedimento. A atuação do enfermeiro nos processos de gerenciamento de recursos materiais e humanos garante a eficácia da assistência de Enfermagem na realização da LECO. Os cuidados de Enfermagem contemplam todas as necessidades fisiológicas e psicossociais, respeitando as individualidades de cada paciente e atentando-se às possíveis complicações pertinentes à recuperação, prestando uma assistência de qualidade durante a permanência dos pacientes do preparo à sala de recuperação anestésica, prezando pela segurança na prevenção dos riscos previsíveis de modo que o paciente viva este momento sem sofrer nenhum dano à sua saúde.

\section{REFERÊNCIAS}

1. ABDELMOWLA RAA, et al. Impact of nursing interventions and patients education on quality of life regarding renal stones treated by percutaneous nephrolithotomy. Journal of Nursing Education and Practice, 2017; 7(12).

2. ACOSTA AM, et al. Atividades do enfermeiro na transição do cuidado: realidades e desafios. Rev. enferm. UFPE on line, 2018; 12(12): 3190-3196.

3. ALVES NG, et al. Complicações no tratamento de urolitíase devido a efeitos adversos da radioterapia pélvica. Revista Eletrônica Acervo Saúde, 2020; 12(9): e3594.

4. Associação Brasileira de Enfermeiros de Centro Cirúrgico, Recuperação Anestésica e Centro de Material e Esterilização (SOBECC). Diretrizes de prática em enfermagem cirúrgica e processamento de produtos para a saúde. Associação Brasileira de Enfermagem de Centro Cirúrgico, Recuperação Anestésica e Centro de Material e Esterilização.7. ed. rev. e atual. Barueri, SP : Manole ; São Paulo, 2017.

5. BENGIO RG, et al. Predictive score of success adapted to our environment to improve results of extracorporeal lithotripsy. Arch Esp Urol. 2016; 69(7): 398-404.

6. BERBER-DESEUSA A, et al. Factores predictores de éxito en litotripsia extracorpórea por ondas de choque (LEOCH). Rev Mex Urol. 2017; 77(4): 251-257.

7. CASTANHEIRA JS, et al. Percepção do paciente no período perioperatório em relação à assistência prestada no centro cirúrgico. Research, Society and Development, 2020; 9(11): e969119573.

8. CAVERZAN R, et al. Humanização no processo de informações prestadas aos acompanhantes dos pacientes cirúrgicos. Arquivos de Ciências da Saúde, 2017; 24(4): 37-41.

9. CONTRERAS PN. Litotricia por ondas de choque para el tratamiento de la litiasis urinaria. Vigencia y efectividad en 2018. Rev Arg Urol. 2018; 83(1): 3-4.

10. FASSARELLA CS, et al. Safety culture at the university surgery center. Research, Society and Development,2020, 9(8): e119985164.

11. FELICI EM, et al. Can renal stone size and the use of the nephrolithometric system increase the efficacy of predicting the risk of failure of percutaneous nephrolithotripsy?. Revista do Colégio Brasileiro de Cirurgiões. 2017; 44(6): 619625.

12. FORNAZARI VAV, et al. Endovascular treatment of abdominal aortic rupture after percutaneous lithotripsy. Radiologia Brasileira. 2021; 54(2): 136-137. 
13. GUTIERRES LS, et al. Good practices for patient safety in the operating room: nurses' recommendations. Rev Bras Enferm. 2018; 71(6): 2775-82.

14. HENRIQUES AHB, et al. Assistência de Enfermagem na Segurança do Paciente Cirúrgico: Revisão Integrativa. Cogitare Enferm. 2016; 21(4): 01-09.

15. JOST MT, et al. Sistematização da assistência de enfermagem perioperatória:avaliando os processos de trabalho no transoperatório. Enfermagem em Foco. 2020; 10(7).

16. KIM JK, et al. Clinical nomograms to predict stone-free rates after shock-wave lithotripsy: Development and internalvalidation. PloS One. 2016; 11(2): e0149333.

17. RODRÍGUEZ GY, et al. Extracorporeal lithotripsy for the treatment of renoureteral lithiasis in the child. RCU. 2019; 8(2): 175-186.

18. SOUZA IB, et al. Percepção do cliente no perioperatório sobre o cuidado de enfermagem no centro cirúrgico. Revista Eletrônica Acervo Saúde. 2019; 26: e840.

19. TANNURE MC, PINHEIRO AM. SAE: Sistematização da Assistência de Enfermagem: guia prático. 2.ed. Rio de Janeiro: Guanabara Koogan; 2010.

20. TORRICELLI FCM, et al. Extracorporeal shock wave lithotripsy in the treatment of renal and ureteral stones. Rev Assoc Med Bras. 2015; 61(1): 65-71.

21. TURK C, et al. EAU Guidelines on Urolithiasis. 2017; 18 (20): 33-35. 